\title{
ANN Model to predict the bake hardenability of Transformation-Induced Plasticity steels
}

\author{
A. Barcellona, D. Palmeri \& R. Riccobono \\ DTMPIG Università degli Studi di Palermo, Italy
}

\begin{abstract}
Neural networks are useful tools for optimizing material properties, considering the material's microstructure and therefore the thermal treatments it has undergone. In this research an artificial neural network (ANN) with a Bayesian framework able to predict the bake hardening and the mechanical properties of the Transformation-Induced-Plasticity (TRIP) steels was designed. The forecast ability of the ANN model is achieved taking into account the operating parameters involved in the Intercritical Annealing (IA), in the Isothermal Bainite Treatment (IBT) and also considering the different prestrain values and the volume fraction of the retained austenite before the Bake Hardening (BH) treatment. This approach allowed one to overcome the need to know the metallurgical rules that describe all the active phenomena in multiphase steels. The neural network approach allowed one to overcome the lack of prediction capability in the existing numerical models.

Keywords: bake hardening, Transformation-Induced Plasticity, neural network, Bayesian framework.
\end{abstract}

\section{Introduction}

The increasing demand for the reduction of automobile $\mathrm{CO}_{2}$ emissions for environmental preservation has lead the automotive industries towards the weight reduction of mechanical components. The main focuses of the automotive market are, indeed, to guarantee safety and comfort while maintaining the light weight of the cars. The Transformation-Induced Plasticity (TRIP) steels allowed one to achieve these goals.

TRIP steels have a multiphase microstructure composed of a ductile ferrite matrix, hard bainite, hard martensite, and retained austenite in metastable 
conditions. The volume fraction of the retained austenite is the origin of the so called TRIP effect that consists of the increasing elongation and strength capability of material produced from the transformation of the retained austenite to martensite under mechanical loading conditions. In order to obtain a large amount of retained austenite, the material is subjected to two thermal treatments, called respectively Intercritical Annealing (IA) and Isothermal Bainite Treatment (IBT).

Another important objective in the development of automotive steel is to reach a good combination of strength and formability. Formability is required when the sheet is shaped into an automobile body panel, whereas high strength is required after assembly. Bake-hardenable steel sheet was developed by exploiting the occurrence that these two properties are not simultaneously needed. After the manufacturing and assembly processes of a car body component, painting and baking are carried out. These processes involve heating the steel body panels to about $443^{\circ} \mathrm{K}$ and maintaining it at this temperature for 30 minutes. At this temperature, the carbon atoms dissolved in the steel diffuse, segregating in the regions around dislocations where the stresses are compressive. This results in a locking of the dislocations, which is called strain aging. This mechanism makes the steel panels harder after baking than after press forming. The utilization of this bake hardening phenomenon has made it possible to use steel sheet that has good formability during press forming and that can withstand severe working, whereas it is hard and less prone to denting when assembled in the car body.

The experimental characterization of the material response, at different values of the main variables that influence the Bake Hardening (BH) and the mechanical properties of TRIP steels, may be both expensive and time consuming, but the evaluation of these factors is necessary to produce components with the desired properties.

Many constitutive numerical models have been developed to evaluate the mechanical properties or the $\mathrm{BH}$ properties of TRIP steels. As described in the following section, for each proposed constitutive model, it is possible to identify a range of thermo-mechanical parameters in which a lack of fit between the experimental and modelled data appears. Furthermore, the metallurgical complexity of these steel requires one to consider the behaviours of each existing phase and also to translate into mathematical expression the phase interactions developed under thermomechanical cycles.

The artificial neural network (ANN) tool offers a forecasting method that may overcome the lack of fit to numerical models and moreover, is able to model the phase transformations phenomena influenced by strong non linear factors. This approach in addition offers the forecasting capability of a model for two aspects, as the ultimate tensile strength (UTS) and the $\mathrm{BH}$, which are produced from different and complex metallurgical modifications. The literature researches offer some neural models able to predict the BH of TRIP steels that start from the chemical composition of material. In this research the capability to take in to account the material variability at the volume fraction retained austenite parameter is assigned (Barcellona et al. [1], Wasilkowska et al. [2], Girault et al. [3].) 
The aim of the conducted study is to develop a model that is able to predict the mechanical strength and the BH effect of TRIP steels as a function of the main parameters that are influent in the three treatments, IA, IBT and BH, by means of a neural network approach with a Bayesian framework. Several published data on the microstructural composition, $\mathrm{BH}$ and mechanical properties of TRIP steels related to the times and temperatures for performing the above mentioned thermal treatments, have been joined with the authors' experimental data in order to define a significant training and validation data set.

\section{TRIP and BH effects}

The designing activity for a forecast tool for the mechanical and functional properties of multiphase materials, such as TRIP steel, needs to start from a deep knowledge of the main metallurgical aspects that affect the $\mathrm{BH}$ and the mechanical properties of TRIP steels both in the case in which the phenomenological approach is considered and in the case of a neural model. The following summarizes the main metallurgical aspects characterizing the TRIP and $\mathrm{BH}$ effects.

\subsection{TRIP effect}

TRIP steels are characterized by a very low content of alloying elements, such as in the tested material the total content of alloying elements is about $3.3 \mathrm{wt}$. pct; in particular $\mathrm{C}, \mathrm{Mn}, \mathrm{Si}, \mathrm{Al}$ are present and other residual elements ( $0.8 \mathrm{wt}$. pct).

For a given chemical composition, the presence of the trip effect is produced from the two-stage heat treatment after cold rolling: the IA and IBT treatments. Considering that carbon is one of the stronger austenite stabilizer elements, the amount of the austenite phase at room temperature in a metastable condition is connected to the austenitic carbon content reached during the Isothermal Bainite Treatment (IBT). In fact, the IBT is the most critical stage of the production process for any TRIP steel. During the IBT, the carbon, which cannot produce the carbides typical of the bainite phase because of the silicon presence, diffuses into the austenitic regions and leads to the stability of the retained austenite at room temperature. The final amount of retained austenite depends therefore on the holding time during the IBT step without the carbide precipitation phenomena and also on the Si content. The silicon alloying also determines the ferrite matrix strengthening by means of solid solution.

During the martensitic transformation of the retained austenite upon mechanical loading, the regions surrounding the transformed phase, in order to accommodate the deformation produced by the phase transformation, undergo a plastic deformation that is added to the deformation produced from the mechanical load. The understanding of relationships between microstructure and mechanical properties requires the analysis of different phase roles.

The TRIP effect arises from the strain-induced transformation of retained austenite to martensite; this transformation result is accompanied by a volume expansion that generates plastic deformation and work hardening of the 
surrounding ferrite phases. In fact, during the first phase of the deformation process, the hard phases dispersed in soft ferrite, i.e. bainite and thermal activated martensite, produce an increasing of the density of dislocations and therefore a high initial value of the work hardening rate. This phenomenon determines a high initial slope in the flow stress curve of the material. Furthermore, in TRIP steels, during the whole deformation process, at increasing of the strain level, the retained austenite progressively transforms itself to the martensite. This phenomenon determines a high work hardening rate and therefore flow stress curve slope; this is also the case for higher strain values. The persistence of the high work hardening rate may be attributed to the formation of stress induced martensite and the accumulation of dislocations in the soft ferrite matrix. Therefore, this strain-induced transformation determines high uniform elongation and also high strength of the material; furthermore it delays the onset of necking and increases the crash energy absorption capability of the material. Finally, the martensitic transformation generates inside the material a compression stress that confers high fatigue resistance (Kumar Srivastava et al. [4], Wang et al. [5]).

\subsection{BH effect}

The BH phenomenon consists of the increasing of yield properties of material after the paint baking treatment. The deforming process of working parts is always experimentally simulated by prestrain. The BH properties are therefore evaluated considering the difference between the yield stress after baking and the flow stress corresponding to a selected percentage of prestrain before the $\mathrm{BH}$ treatment. This treatment consists of the aging of the material at $443^{\circ} \mathrm{K}$ for 30 minutes. Many factors, such as the bainite phase-transformation, increasing of the carbon content in the retained austenite, decreasing of the retained austenite content and increasing of the dislocation density in ferrite matrix, influence the yielding phenomenon of tensile prestrained and baked trip steel sheets.

It is possible to distinguish different contributions inside the yield variation connected to the paint baking treatment. Initially there is an increment of yield stress produced into the ferrite matrix due to an activate diffusion of the solid solute, which determines a hindrance of dislocation movement. Upon longer aging times, carbides precipitate out the $\mathrm{C}$ atmospheres around the dislocations, resulting in an increase in both yield stress and ultimate tensile strength. Another contribution of yield increment is produced from increasing of the carbon content in the retained austenite during baking and the produced solid solute strengthening effect determines the strength of the retained austenite. It also needs to be remarked that during the baking treatment, a decrease in the amount of the retained austenite due to the austenite transformation to bainite, which is more stable at the given temperature, appears. Each contribution on the yield properties of material is connected on the amount of each phase. During the BH treatment the amount and therefore the yield contribution of the martensitic phase does not change. 


\section{The existing numerical models}

The existing numerical models consider these particular multiphase steels as a composite material, and therefore start from the phenomenological laws that describe the mechanical behaviour of each existing phase. The interaction between the martensite and austenite phases is examined using the Gladman-type mixing law and foresees the assumption of the partition stress and strain mechanism between the two phases. The kinetics of the strain induced transformations are modelled using the Olson-Cohen equations. This approach allows one to simulate the mechanical behaviours of TRIP steels, but does not consider the main aspect connected to the BH effect, and therefore the results are unable to predict this important aspect of TRIP steels. The other most used numerical models to simulate the mechanical behaviours of TRIP steels are the Johnson-Cook, the Ludwig and the Zhao models or variants of these. Each numerical model has a specific applicability range that is coupled with the determination of strain ranges in which the fitting capability of the model decreases. These models also do not consider the physical aspect connected to the BH effect (Shan et al. [6], Liu et al. [7], Li et al. [8], Bouquerel et al. [9]).

\section{The ANN technique}

The ANN approach constitutes a regression analysis method in which a flexible non linear function is fitted to the experimental data. This tool is able to capture complex relationships characterizing phase transformations, without requiring mathematical descriptions of phenomena.

The Bayesian framework applied to the neural model is able to take into account the fitting uncertainty. This method calculates a probability distribution of the set of neural network weights and provides the outputs error bars, defining the applicability range of the neural model. Furthermore, the significance of the input variable is automatically quantified.

Considering the Kolmogorov theorem, the complexity of each system can be captured with a neural network model containing a single hidden layer; the flexibility of the model is attained operating on the number of the hidden units. The general model formulation considering a feed-forward architecture with one hidden layer and $\mathrm{i}$ hidden units is:

$$
y_{k}=\sum_{i} w_{k i}^{(2)} h_{i}+\theta_{k}^{(2)},
$$

where

$$
h_{i}=\tanh \left(\sum_{j} w_{i j}^{(1)} x_{j}+\theta_{i}^{(1)}\right) \text {, }
$$

$x_{j}$ are the inputs, $y_{k}$ are the outputs, $\theta$ are the bias corresponding to each neural node, $w$ are the neural weights and the superscript (1) refers to the hidden layer, whereas the superscript (2) refers to the output layer. Eqn (1) expresses the output of the neural model, whereas eqn (2) expresses the transfer function. The 
combination of several hyperbolic tangents confers to the model the ability to capture the non linear relationship between inputs and outputs.

The number of input, output and hidden nodes and their connections defines the architecture of the neural model. The Bayesian framework foresees that the weights and biases of the network are assumed to be random variables with specified distributions and provides a method to improve the generalization capability of the neural network, usually called regularization.

The backpropagation algorithm is able to train multilayer feed-forward networks with differentiable transfer functions to perform function approximation, pattern association, and pattern classification. There are several backpropagation training algorithms; among them, the Bayesian regularization algorithm consists of a modification of the Levenberg-Marquardt training algorithm to produce networks that generalize well, reducing the difficulty of determining the optimum network architecture.

The Bayesian regularization involves modifying the performance function, which normally is the sum of the squares of the network errors on the training set. The formulation of the Bayesian performance function is depicted in eqn (3):

$$
M S E_{\text {reg }}=\gamma M S E+(1-\gamma) M S W
$$

in which $M S E_{\text {reg }}$ is the modified performance function, $\gamma$ is the performance ratio, $M S E$ is the typical performance function mean squared error given by:

$$
M S E=\frac{1}{N} \sum_{i=1}^{N}\left(e_{i}\right)^{2}=\frac{1}{N} \sum_{i=1}^{N}\left(t_{i}-y_{i}\right)^{2},
$$

in which $t_{i}-y_{i}$ represents the difference between the target value and the output value and $M S W$ is the mean of the sum of the squares of the network weights:

$$
M S W=\frac{1}{n} \sum_{i=1}^{n}\left(w_{j}\right)^{2}
$$

The determination of the optimum value for the performance ratio parameter $(\gamma)$ allows one to generate a network that best fits the training data. In effect, if this parameter is too large, it may get overfitting and if the ratio is too small, the network will not adequately fit the training data.

The described network architecture has been implemented using the MATLAB neural network toolbox that provides some routines that automatically set the regularization parameter. The Bayesian regularization works well if the input and the target data are ranged in $[-1 ; 1]$. Therefore, the inputs and the targets have been normalized within the range $[-1 ; 1]$ before training as follows:

$$
x_{\text {norm }}=2 \frac{x-x_{\min }}{x_{\max }-x_{\min }}-1 \text {, }
$$

where $x_{\text {norm }}$ is the normalized value of each parameter and $x, x_{\min }$ and $x_{\max }$ are respectively the measured, the minimum and the maximum values for the 
considered parameter. In order to avoid the possibility of overfitting data, the experimental data are randomly divided into two groups respectively called the training set and the test set. The model has been implemented considering the only training data set constituted by 75 pet of the experimental data and it has been tested considering the test data set constitute by 25 pct of the experimental data (De Cooman [10], Cetinel et al. [11], Dobrzanski and Trzaska [12], Garcia et al. [13], Das et al. [14]).

\section{Model application and results}

The topological definition of the selected neural model started from the individuation of the main parameters that influence the selected forecasting outputs: the $\mathrm{BH}$ index and the UTS value. The strong influence of the two thermal treatments able to confer to the material the TRIP effect was considered, selecting as input of the neural network the temperatures and the times of both the IA treatment and the IBT treatment. The paint baking treatment was taken into account, choosing as an input the prestrain value, considering that the $\mathrm{BH}$ index is a function of the selected prestrain level. The operating parameters of the paint baking treatment were considered as constant and equal to $443^{\circ} \mathrm{K}$ and 30 minutes. The variability of the chemical composition of steel was considered, inserting among the input the volume fraction of the retained austenite achieved after the IA end the IBT treatments. Each designed neural model therefore has six input parameters, as depicted in table 1 . The considered outputs were the $\mathrm{BH}$ index and the UTS value. The Bayesian regularization used gives good results if the input and the target data are ranged in $[-1 ; 1]$; for this reason, the inputs and the targets have been normalized, highlighting the maximum and the minimum values of each considered input and output.

The fitting capability of the neural model was investigated, designing different neural networks containing a variable number of the hidden units.

Table 1: $\quad$ Variation ranges of input and output parameters.

\begin{tabular}{|c|l|c|c|}
\hline \multicolumn{2}{|c|}{} & Max & Min \\
\hline \multirow{4}{*}{ Inputs } & $\mathrm{T}_{\text {IA }}\left({ }^{\circ} \mathrm{K}\right)$ & 1086 & 1031 \\
\cline { 2 - 4 } & $\mathrm{t}_{\mathrm{IA}}(\mathrm{s})$ & 600 & 120 \\
\cline { 2 - 4 } & $\mathrm{T}_{\mathrm{IBT}}\left({ }^{\circ} \mathrm{K}\right)$ & 733 & 643 \\
\cline { 2 - 4 } & $\mathrm{t}_{\mathrm{IBT}}(\mathrm{s})$ & 960 & 120 \\
\cline { 2 - 4 } & Prestrain $(\mathrm{pct})$ & 20 & 0 \\
\cline { 2 - 4 } & $\mathrm{V}_{\text {ret }}(\mathrm{pct})$ & 16 & 4 \\
\hline \multirow{3}{*}{ Outputs } & \multicolumn{3}{|c|}{} \\
\hline & $\mathrm{BH}(\mathrm{MPa})$ & 80 & 50 \\
\cline { 2 - 4 } & $\mathrm{UTS}(\mathrm{MPa})$ & 1050 & 790 \\
\hline
\end{tabular}


The selection of the best number of the hidden neurons has been made using a pruning algorithm that uses sensitivity analysis to quantify the relevance of inputs and hidden units proposed by Engelbrecht [15]. The obtained best performance allowed one to select the model with a complexity level able to optimize the forecasting capability of the neural network. The set of data useful for defining the training and the testing of each neural model were collected considering a wide experimental campaign, previously conducted, to highlight the influence of the IA and IBT operating parameters on the UTS and the BH properties of TRIP steel (Barcellona et al. [16, 17]). In order to enlarge the training and the test data set, experimental results also derived from literature data have been considered (Zhang et al. [18], Timokhina et al. [19], Wang et al. [20], Pereloma et al. [21], Kvačkaj and Mamuzić [22]). In order to highlight the trend of performance data at the varying of the number of the hidden neurons, eight neural networks differing in the number of the hidden neurons have been displayed in their training and testing phases. The detailed topology of each neural network is reported in table 2, in which the performance results are also summarized in terms of the correlation coefficient $\mathrm{R}$ between the forecasted and the experimental outputs.

In order to achieve a better readability of the obtained results, the outputs of each neural network have been post-processed; the linear regression between the network response and the target data allowed one to evaluate the fitting capability of the model to the experimental data in the training and in the testing phases. The evaluation of the regression coefficient $\mathrm{R}$ provided the degree of correlation between the experimental and the foreseen data.

Twenty-two hidden units offers a sufficient complexity level to best fit the experimental data. In effect, the observation of the values of the R coefficient of two outputs in the training phase evidenced that a lower number of hidden units is insufficient to best fit the experimental data, but the fitting capability increases with the increasing of the hidden neurons and it attains the maximum in the neural network model 6-22-2.

Table 2: $\quad$ Outputs correlation coefficients $\mathrm{R}$ of the training and the testing phases.

\begin{tabular}{|l|c|c|c|c|}
\hline $\begin{array}{l}\text { Neural } \\
\text { Networks }\end{array}$ & $\mathrm{R}_{\text {training }} \mathrm{BH}$ & $\mathrm{R}_{\text {testing }} \mathrm{BH}$ & $\mathrm{R}_{\text {training }} \mathrm{UTS}$ & $\mathrm{R}_{\text {testing }} \mathrm{UTS}$ \\
\hline $6-5-2$ & 0.495 & 0.397 & 0.523 & 0.497 \\
\hline $6-7-2$ & 0.872 & 0.823 & 0.891 & 0.823 \\
\hline $6-10-2$ & 0.883 & 0.842 & 0.918 & 0.882 \\
\hline $6-14-2$ & 0.885 & 0.844 & 0.917 & 0.885 \\
\hline $6-20-2$ & 0.919 & 0.897 & 0.920 & 0.902 \\
\hline $6-22-2$ & 0.944 & 0.943 & 0.965 & 0.963 \\
\hline $6-24-2$ & 0.943 & 0.932 & 0.958 & 0.950 \\
\hline $6-27-2$ & 0.921 & 0.914 & 0.928 & 0.915 \\
\hline
\end{tabular}



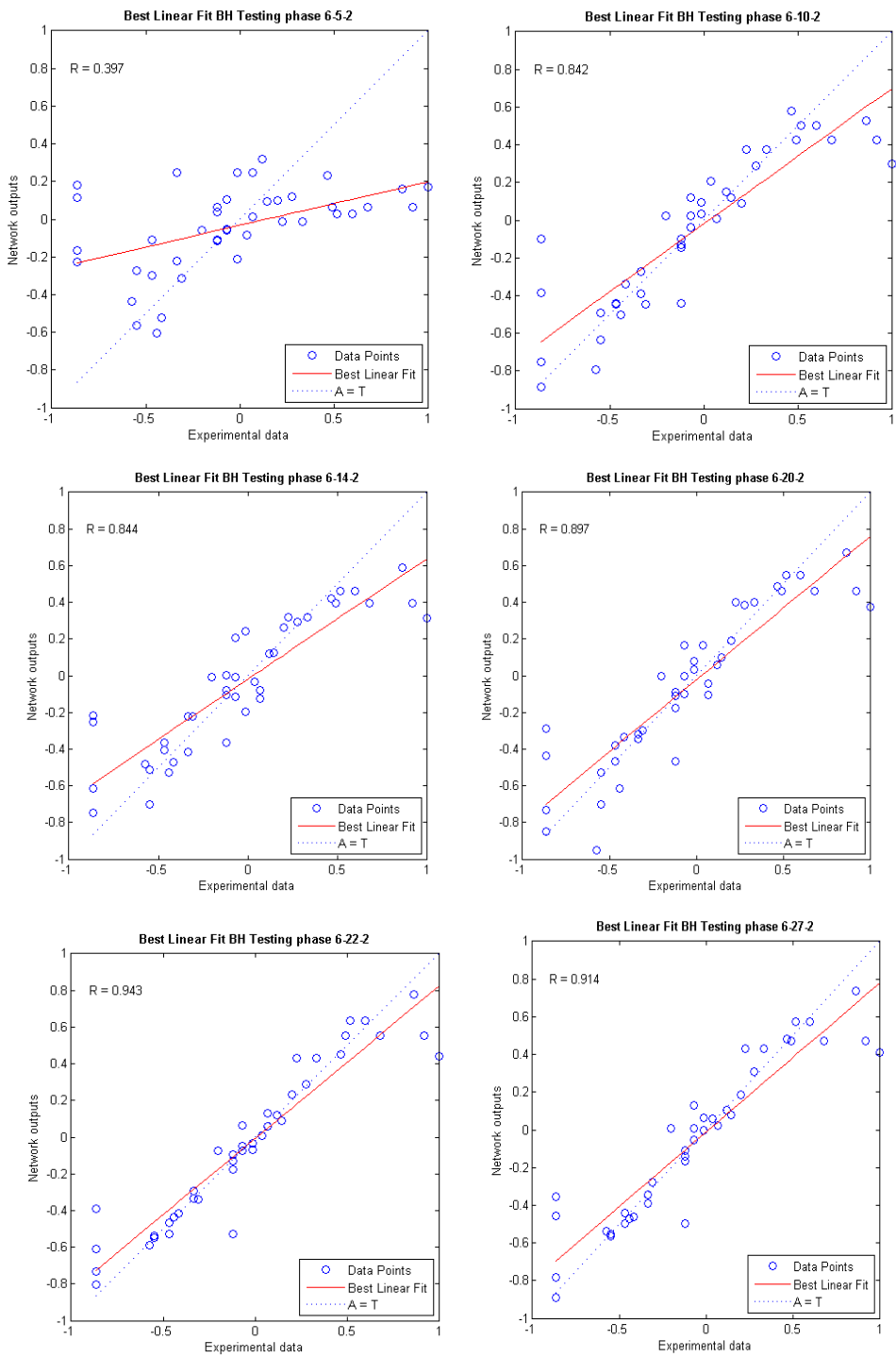

Figure 1: Linear regression between the network response and the target in the testing phase for the $\mathrm{BH}$ output parameter.

This optimum of performances was found for both the considered outputs. The interpolation capability of the forecasting tool was also investigated in the testing phase by the evaluation of the performance coefficient $\mathrm{R}$; a light decreasing of the fitting capability in respect to the training phase has been observed. 
This difference becomes almost pointless for the best neural model. This tendency has been observed for both outputs.

The designed neural model shows better forecast ability for the UTS values, but the difference in the $\mathrm{R}$ values of the two output parameters tends to decrease when the number of neurons in the hidden layer approaches the best number of hidden neurons.

The image visualization of the results of the regression analysis in the testing phase, as depicted in figure 1, allowed one to evaluate how the forecasted data differs from the experimental data, and it directly shows the dispersion effect produced by the neural model in respect to the best fitting condition, represented by the $45^{\circ}$ inclined line.

\section{Conclusions}

The main focuses of the automotive market are to guarantee safety and comfort while maintaining the light weight of the cars and preserving a good combination of strength and formability. These goals are achieved by the development of TRIP steels that posses the BH effect. In this research an ANN with a Bayesian framework able to predict the $\mathrm{BH}$ and the mechanical properties of the TRIP steels was designed. The neural approach allowed one to overcome the lack of prediction capability of the existing numerical models. The main obtained results are summarized as follows.

- The selection of the best number of the hidden neurons has been made using a pruning algorithm that uses sensitivity analysis to quantify the relevance of input and hidden units proposed by Engelbrecht.

- In order to highlight the trend of performance data at the varying of the number of the hidden neurons, eight neural networks differing in the number of the hidden neurons have been displayed in their training and testing phases.

- The outputs of each neural network has been post-processed; the linear regression between the network response and the target data allowed one to evaluate the fitting capability of the model to the experimental data in the training and in the testing phases.

- The observation of the values of the $\mathrm{R}$ coefficient of the outputs in the training phase evidenced that a low number of hidden units is insufficient to best fit the experimental data, but the fitting capability increases with the increasing of the hidden neurons and reaches the maximum in the neural network model 6-22-2. This optimum of performances was found for both the outputs.

- The interpolation capability of the forecasting tool was investigated by the evaluation of the performance coefficient $\mathrm{R}$ in the testing phase. A light decreasing of the fitting capability in respect to the training phase has been observed. This difference becomes almost pointless for the best neural model and this tendency has been observed for both the outputs. 
- The designed neural model possesses better forecast ability for the UTS values, but the difference in the $R$ values of the two output parameters tends to decrease when the number of the hidden units approaches the best number of hidden neurons.

- The graphic visualization of the results of the regression analysis allowed one to evaluate how the forecasted data differ from the experimental data and the degree of dispersion from the best fit condition.

\section{References}

[1] Barcellona A, Palmeri D. Multi-Layer Neural Network Application for Optimization of Thixotropic Aluminium Alloy Process Parameters. In: Intelligent Computation in Manufacturing Engineering 5. 5th CIRP ICME. Ischia, Italy, (pp. 139-144). ISBN/ISSN: 8895028015 - 97888 $950280,2006$.

[2] Wasilkowska, Tsipouridis P., Werner E.A., Pichler A., Traint S., Microstructure and tensile behaviour of cold-rolled TRIP-aided steels, Journal of Materials Processing Technology 157-158, 633-636. 2004.

[3] Girault E., Mertens A., Jacques P., Houbaert Y., Verlinden B., Van Humbeeck J., Comparison Of The Effects Of Silicon And Aluminium On The Tensile Behaviour Of Multiphase Trip-Assisted Steels, Scripta mater. 44, 885-892. 2001.

[4] Kumar Srivastava A., Jha G., Gope N., Singh S.B., Effect of heat treatment on microstructure and mechanical properties of cold rolled CMn-Si TRIP-aided steel, Materials Characterization 57, 127-135. 2006.

[5] Wang X.D., Huang B.X., Rong Y.H., Wang L., Microstructures and stability of retained austenite in TRIP steels, Materials Science and Engineering A, 438-440, Pages 300-305, 2006.

[6] Shan T.K., Li S.H., Zhang W.G., Xu Z.G., Prediction of martensitic transformation and deformation behaviour in the TRIP steel sheet forming, Materials and Design 29 1810-1816, 2008.

[7] Liu Jun-Yan, Lu Hao, Chen Jun-Mei, Jullien J. F., Wub Tong, Simulation of mechanical behaviour of multiphase TRIP steel taking account of transformation-induced plasticity, Computational Materials Science $\mathbf{4 3}$ 646-654, 2008.

[8] Li S.H., Dan W.J., Zhang W.G., Lin Z.Q., A model for strain-induced martensitic transformation of TRIP steel with pre-strain, Computational Materials Science 40 292-299, 2007.

[9] Bouquerel J., Verbeken K., De Cooman B.C., Microstructure-based model for the static mechanical behaviour of multiphase steels, Acta Materialia, 54, 1443-1456. 2006.

[10] De Cooman B.C., Structure-properties relationship in TRIP steels containing carbide-free bainite, Current Opinion in Solid State and Materials Science, 8, 285-303, 2004. 
[11] Cetinel H., Özyiğit H.A., Özsoyeller L., Artificial neural networks modelling of mechanical property and microstructure evolution in the Tempcore process, Computers and Structures 80, 213-218. 2002.

[12] Dobrzanski Leszek A., Trzaska J., Application of neural networks for the prediction of continuous cooling transformation diagrams, Computational Materials Science 30, 251-259. 2004.

[13] Garcia-Mateo Carlos, Capdevila Carlos, Caballero Francisca Garcia, Garcia de Andres Carlos, Artificial neural network modelling for the prediction of critical transformation temperatures in steels, J Mater Sci, 42, 5391-5397, 2007.

[14] Das S., Singh S. B., Mohanty O. N. and Bhadeshia H. K. D. H., Understanding the complexities of bake hardening, Materials Science and Technology Vol $24 \mathrm{~N}^{\circ}$ 1, pp. 107-111, 2008.

[15] Engelbrecht A. P., A New Pruning Heuristic Based on Variance Analysis of Sensitivity Information, IEEE TRANSACTIONS ON NEURAL NETWORKS, VOL. 12, NO. 6, November 2001.

[16] Barcellona A, Cannizaro L, Palmeri D., Microstructural Characterisation of Thermo-Mechanical Treated Trip Steels. Key Engineering Materials. Vol. 344, Pp. 71-78 ISSN: 1013-9826. 2007.

[17] Barcellona A, Cannizzario L, Palmeri D., Effect of intercritical annealing and of isothermal bainite treatment on microstructure, mechanical and bake hardening properties of TRIP 800 steel, Proceedings of $8^{\text {th }}$ AITeM conference. (pp. 73-74). ISBN/ISSN: 88-7957-264-4. 2007.

[18] Zhang J., Fu R., i Zhang M., Liu R., Wei X. and Li L., Bake hardening behaviour of TRIP and DP steels, Journal of University of Science and Technology Beijing Volume 15, Number 2, Page 132, 2008.

[19] Timokhina I.B., Hodgson P.D., and Pereloma E.V., Transmission Electron Microscopy Characterization of the Bake-Hardening Behaviour of Transformation-Induced Plasticity and Dual-Phase Steels, Metallurgical and Materials Transactions A, 2442-Volume 38a, October 2007.

[20] Wang Z.C., Kim S.J., Lee C.G., Lee T.H., Bake-hardening behaviour of cold-rolled CMnSi and $\mathrm{CMnSiCu}$ TRIP-aided steel sheets, Journal of Materials Processing Technology, 151, 141-145. 2004.

[21] Pereloma E.V., Russell K.F., Miller M.K. and Timokhina I.B., Effect of pre-straining and bake hardening on the microstructure of thermomechanically processed CMnSi TRIP steels with and without $\mathrm{Nb}$ and Mo additions, Scripta Materialia 58, 1078-1081, 2008.

[22] Kvačkaj T., Mamuzić I., Development Of Bake Hardening Effect By Plastic Deformation And Annealing Conditions, METALURGIJA 45 1, 51-55, 2006. 